\title{
XXXIX. The density of the æther
}

\section{Sir Oliver Lodge}

To cite this article: Sir Oliver Lodge (1907) XXXIX. The density of the æther , Philosophical Magazine Series 6, 13:76, 488-506, DOI: 10.1080/14786440709463624

To link to this article: http://dx.doi.org/10.1080/14786440709463624

曲 Published online: 16 Apr 2009.

Submit your article to this journal 정

Џll Article views: 9

Q View related articles $\asymp$

Citing articles: 2 View citing articles 
suitable density. This density is matched by rotation of the second nicol before and after the interposition of the substance under test. The difference of readings gives, of course, the optical rotation for the part of the spectrum under test.

In the instrument exhibited room is provided for the interposition of a $100 \mathrm{~mm}$. tube for liquids, but there is of course no difficulty in extending this to any desired amount. It will be seen that one measures by reducing one half of the field to the intensity of the other half which is already fairly dark. Thus one would expect the sensitiveness to be somewhat less than that obtained by the use of polarizers, in which one half of the field decreases while the other half increases in intensity. But it is an exceedingly useful addition to the spectrophotometer and produces a polarimeter free from some serious defects of most instruments excepting those with the Lippisch and similar polarizers. Moreover it is, of course, suitable either for use by monochromatic light or for the measurement of optical rotations for different wave-lengths.

I may add that a photographic negative is the best neutraltint medium with which I am acquainted, and is what is employed when using the instrument as a spectro-polarimeter.

\section{The Density of the Ether. By Sir OLivfr LODGE *. Part I.}

1. THE ætherial constants, $\mu$ and $\kappa$,- the magnetic permeability and the electric inductivity of free space, have hitherto been of completely unknown value, both as to kind of quality and as to numerical magnitude; though the reciprocal of their geometric mean is well known to be a velocity, the velocity with which the rther transmits transverse waves of all lengths and intensities.

2. For many reasons I have been accustomed to think of $4 \pi \mu$ (the ungeometrical part of a coefficient of self-inductance) as of something which by legitimate analogy could be spoken of as æetherial density, and of $4 \pi / \kappa$ as of something which by similar material analogy must be expressible as ætherial elasticity or rigidity: this kind of rigidity being probably due to the æther's own intrinsic energy of constitution, and being explicable on a recondite hydrodynamical basis, as treated in various forms by Lord Kelvin.

3. I have also adduced reasons (in 'Modern Views of Electricity') for regarding the ther as exceedingly, perhaps infinitely, incompressible; this view being based in the first

* Communicated by the Author. 
instance on the "superficial-charge" experiment of Cavendish (carefully repeated by Clerk Maxwell and the present Principal of Glasgow University with modern appliances) *; and in the second instance on the phenomenon of gravitation.

4. Furthermore, of late years, the whole world bas become accustomed to think of minute electric charges-configurations probably of æther of some kind-moving about freelr, and endowed with a definite mass and bulk: and, as is well known, the hypothesis has been mooted that matter itself may consist of bodies of this sort of character.

But if so, it is apparent how very porous, and so to speak rarefied, a structure matter must exhibit, to every thing except electric disturbance; for the interstices must be enormous compared with the regions of definite structure. So, just as the ratio of mass to volume is small in the case of a solar system or a nebula or a colbweb, I have been driven to think that the observed mechanical density of matter is probably an excessively small fraction of the total density of the substance, or æther, contained in the space which it thus partially occupies - the substance of which it may hypothetically be held to be composed.

5. Thus, for instance, consider a mass of platinum, and assume that its atoms are composed of electrons, or of some structures not wholly dissimilar: the space which these bodies actually fill, as compared with the whole space which in a sense they 'occupy,' is comparable to $10^{-10}$ th of the whole, even inside each atom; and the fraction is still smaller if it refers to the visible mass. So that a kind of minimum estimate of ætherial density, on this basis, would be something like ten-thousand-million times that of platinum.

This, standing alone, would be a wild guess; but other physicists have probably of late years been led, on various grounds, to suspect that the etherial density may be large ; though probably it has not occurred to them to surmise that it is as great as that. Nevertheless if more detailed consideration should urge us to contemplate a density of enormous magnitude for the æther, even greater than the above, there is no need to be shocked or surprised.

6. With this introduction I will state the argument as briefly as possible ; referring for fuller or more explanatory details to the concluding chapter of a new edition of 'Modern Views of Electricity':-

* Maxwell, 'Cavendish Researches,' p. 104, and Note 19, p. 417. See also Proc. Camb. Phil. Soc. vol. ii. part iii,, and Maxwell's Collected Works, p. 612. 
It is admitted that each negative electron has a certain intrinsic inertia or massiveness, dependent upon the amount of electricity which constitutes it, and on its size when pictorially represented as like a charged sphere; the mass being constant for all ordinary ranges of speed.

The simplest way of illustrating such a mass is to treat it as if it were a sphere of radius a moving through a simple irrotational perfect liquid, of density $\rho$, which it merely displaces.

I'the ather being supposed incompressible, as stated above, the density inside and outside the sphere must, on the simplest hypothesis, be assumed the same. And accordingly the total effective mass of the moving body is half as much again as the mass of the sphere itself, viz. $2 \pi \rho a^{3}$; since in such a case the body is known, from ordinary hydrodynamics, to behave as if it carried half its bulk of fluid with it, while all the rest was annihilated.

That is the simplest mode of regarding the matter.

7. But it is known that the apparent mass of an electric charge really resides in the space outside it; being due to the circular magnetism which surrounds its path. If its speed is $u$, the intensity of magnetic field at any place $r \theta$, due to the motion, is, in every system of units,

$$
\mathrm{H}=\frac{e u \sin \theta}{r^{2}} .
$$

[Parenthetically we may here observe, without any hypothesis, how enormous is the magnetic intensity immediately surrounding the equator of an electron, moving along an axis at say $\frac{1}{3}$ th the speed of light. It is no less than $10^{15}$ c.G.s. units. At a third the speed of light, even in the completer theory of which the above is the dominant term at ordinary speeds, it is still not appreciably more than $10^{16}$; but it begins to run up suddenly towards infinity when the velocity of light is closely approached. The reason probably being that it is then no longer a small fraction of the intrinsic rotational energy of the rether itself.]

8. Now mathematical speculation has suggested that there is an æetherial flow or circulation along lines of magnetic induction, which are well known to be always closed curves; and it is natural to assume that the energy of the field may be represented by the energy of this motion. Denote the speed of this hypothetical æether-drift by $w$, then the energy per unit volume is expressible in two equivalent ways, namely,

$$
\text { as } \frac{1}{2} \rho w^{2} \text {, and as } \frac{\mu \mathrm{H}^{2}}{8 \pi} \text {. }
$$


Wherefore, in the case when the field is excited by a single spherical moving charge, $e$,

$$
\frac{w}{u}=\frac{e \sin \theta}{r^{2}} \sqrt{\left(\frac{\mu}{4 \pi \rho}\right) .}
$$

At the equator of the sphere, where $r=a$ and $\theta=90^{\circ}, w$ is a maximum, say $w_{n}$, such that

and so

$$
\frac{w_{0}}{u}=\frac{e}{a^{2}} \sqrt{ } /\left(\frac{\mu}{4 \pi \rho}\right) ;
$$

$$
w_{0}=\frac{a^{2}}{r^{2}} \sin \theta \text {. }
$$

Thus the magnetic circulation round an electron, though certainly intense in its immediate neighbourhood, decreases as the square of the distance; and at any perceptible microscopic interval of space is utterly insignificant.

9. Certain imperfect cog-wheel, or rack-and-pinion, analogies, elsewhere developed-with a screw motion on the axes of the pinions by reason of the gyrostatic structure of the medium-suggest that $w_{0}$ and $u$ are velocities of the same order of magnitude,--suggest even that they may be equal, or connected by the ratio $\pi$.

Now although this is uncertain and hypothetical, yet it is difficult to suppose $w_{0}$ greater than $u$, since it is excited by it ; moreover $u$ is already often so enormous, sometimes nearly equal to the velocity of light. While if $w_{0}$ be regarded as decidedly less than $u$, the density of the æther, shortly to be estimated on this basis, will be still further increased, in proportion to the square of the ratio $u: w_{0}$, to an amount which becomes even more immense. As a working hypothesis, therefore, I propose to assume that $w_{0}$ and $u$ are of the same order of magnitude and practically equal.

10. An immediate consequence of this is that, in every system of units,

$$
e=4 \pi a^{2} \sqrt{\left(\frac{4 \pi \mu}{\rho}\right) ;}
$$

and as there are rensons (indicated in 'Modern Views') for supposing that the quantity under the square root is unity, the electronic charge comes out as equal, both in magnitude and dimensions, to the superficies of its supposed spherical bulk. The ordinary electrostatic unit of charge, moreover, happens to equal the superficies of the conventional atom: and the dimensions of all electrostatic and magnetic quantities are as given in Appendix $p$ of 'Modern Views of Electricity' (1889). 11. But this is a minor matter; let us proceed to estimate 
the massiveness of the hypothetical magnetic circulation, without any assumption of that kind.

Its energy will be

$$
\int_{0}^{\pi} \int_{a}^{\infty} \frac{1}{2} \rho w^{2} \cdot 2 \pi r \sin \theta \cdot r d \theta \cdot d r .
$$

Substituting for $w$ its value just found in $\S \S 8,9$,

$$
w=\frac{a^{q}}{r^{2}} \cdot u \sin \theta,
$$

the term to be integrated becomes

$$
\pi \rho a^{4} u^{2} \cdot \frac{d r}{r^{2}} \sin ^{3} \theta d \theta
$$

and so the energy comes out

$$
\frac{4 \pi \rho a^{4} u^{2}}{3 a} .
$$

Expressing this as the linetic energy of a certain mass $m$ moving with speed $u$, this gives

$$
m=\frac{8}{3} \pi \rho a^{3} ;
$$

or twice the mass of the content of the sphere, considered as composed of the surrounding magnetically circulating medium of density $\rho$. In so far as $w_{0}$ and $u$ are not equal, this expression for $m$ becomes multiplied by the factor $\left(\frac{w_{0}}{u}\right)^{2}$, which justifies the remark of $\S 9$.

12. All the estimates of inertia agree, therefore, as regards order of magnitude :-

(1) The simple sphere composed of the coagulated liquid;

(2) The sphere moving through effectively displaced simple incompressible fiuid ;

(3) The more elaborate and truer consideration of the magnetic circulation outside the sphere.

For they are to one another merely in the ratio

$$
1: 1 \frac{1}{2}: 2 \text {; }
$$

and they all give the effective mass of the electron as due to a disturbance, moving and identified with itself, affecting a region comparable with its own bulk. That region therefore must have a certain density.

What is that density?

We all admit now that the mass of an electron is comparable 
with $10^{-2 \pi}$ gramme, while the volume is comparable with $10^{-39}$ c.c.; so that the density of the medium must be of the order $10^{12}$ grammes per c.c.;

and the absolute value of the magnetic constant $\mu$ for free space will be about $10^{11}$ of the same units.

The electrostatic rigidity of the medium is therefore $10^{33}$ dynes per sq. cm.;

or the absolute value of the dielectric constant $\kappa$ for free space is $10^{-32}$ cubic centimetre per erg.

The intrinsic constitutional kinetic energy of the æther, which confers upon it its properties and enables it to transmit waves, is thus comparable with $10^{83} \mathrm{\epsilon rgs}$ per c.c.;

or say 100 foot-lbs. per atomic volu ne.

This is equivalent to saying that $3 \times 10^{17}$ kilowatt-hours, or the total output of a million-kilowatt power station for thirty million years, exists permanently, and at present inaccessibly, in every cubic millimetre of space.

13. Just as the ætherial rigidity is of a purely electric character, and is not felt mechanically-since mechanically it is perfectly fluid, - so its density is likewise of an electromagnetic character, and again is not felt mechanically, because it cannot be moved by mechanical means. It is by far the most stationary body in existence; though it is endowed with high intrinsic energy of local movement, analogous to turbulence, conferring on it gyrostatic properties.

Optically, its rigidity and density are both felt, since optical disturbances are essentially electromotive. Matter loads the xther optically, in accordance with the recognized fruction $\frac{n^{2}-1}{n^{2}}$; and this loading, being part and parcel of the matter, of course travels with it. But it is not a mechanical loading.

The mechanical density of matter is a very small proportion of the wtherial density; but the optical or electrical density of matter-being really that of æther affected by the intrinsic or constitutional electricity of matter-is not so small. The relative optical virtual density of the æther inside and outside matter is measured by $n^{2}$; but it may be really a defect of elasticity, at least in non-magnetic materials.

Electrical and optical effects may be said to depend upon $e$. Mechanical or inertia effects may be said to depend upon $e^{2}$.

Detached and inelastic charges can load the other optically, quite appreciably; but as regards mechanical loading, the densest matter known is trivial and gossamer-like compared with the unmodified æther in the same space. 


\section{Some consequences of this estimate of Density.}

14. The amplitude of a wave of light, in a place where it is most intense, namely near the sun where its energy amounts to 2 ergs per c.c., comes out only about $10^{-17}$ of the wavelength. The maximum tangential stress called out by such strain is of the order $10^{11}$ atmospheres.

The hypothetical luminous circulation-velocity, conferring momentum on a wave-front, in accordance with Poynting's investigation, comes out $10^{-22} \mathrm{~cm}$. per sec. The arithmetic of these calculations is given in the new concluding chapter of 'Modern Views' already referred to, $\S 6$, which is likely also to appear in 'Nature.'

The supposed magnetic ætherial drift, along the axis of a solenoid or other magnetic field, is comparable to .003 centim. per sec., or 4 inebes an hour, for a field of intensity 12,000 c.c.s.

But it is not to be supposed that this hypothetical velocity is slow everywhere. Close to an electron the speed of magnetic drift is comparable to the locomotion-velocity of the electron itself, and may therefore rise to something near the speed of light; say $\frac{1}{30}$ th of that speed: but in spite of that, at a distance of only 1 millimetre away, it is reduced to practical stagnation, being less than a millimicron per century.

In any solenoid, the ampere-turns per linear inch furnish a measure of the speed of the supposed magnetic circulation along the axis-no matter what the material of the core may be-in millimicrons per sec.

[1 micron $=10^{-6}$ metre, 1 millimicron is $10^{-9}$ metre $=10^{-7}$ centimetre.]

To get up an ætherial speed of 1 centimetre per secondsuch as might be detected experimentally by refined optical appliances, through its effect in accelerating or retarding the speed of light sent along the lines of magnetic force,-would need a solenoid of great length, round every centimetre of which 1000 amperes circulated 3000 times. That is to say, a long field of four million c.G.S. units of intensity.

15. The magnetic circulation, being thus extremely difficult if not hopeless to test experimentally, it may be thought that by sending light down a torrent of cathode rays in a vacuumtube, a better attempt to observe some accelerating or retarding effect could be made; because here a small portion of the xther is carried forward with enormous speed. But the excessive tenuity of the moving portion is against the possibility of detection. It is not the mechanical density of the residual gas, as compared with that of the stagnant æther, that need be considered however; - that would indeed be small, 
being $10^{-6} \times 10^{-3} \times 10^{-12}=10^{-21}$, at the exhaustion of a millionth of an atmosphere, even supposing the whole contents of the tube were electrons,- -but it is the optical density; and that would appear to be something more like $10^{-6}\left(n^{2}-1\right) / n^{2}$, where $n$ is the refractive index for common air. The optical fraction being $\frac{1}{2000}$, this amounts to $\frac{1}{2} \cdot 10^{-8}$; and at a speed of $10^{9} \mathrm{~cm}$. per sec. it would seem to give something almost appreciable in the way of drift. But the torrent of electrons must be only a smallfraction of the whole residual gas; and sume positive charges are sailing up in the other direction, in numbers probably sufficient to compensate for their smaller speed. So the outlook for experiment on this kind of influence of electricity on light is not encouraging,--whether the question be examined from the point of view of magnetic flux, or from that of electric current.

The estimates of this paper are:-That the æther has a magnetic inertia-coefficient which can be represented by likening it to a mechanical medium with a density of $10^{1_{2}}$ grammes per cubic centimetre; that the intrinsic æetherial kinetic energy is $10^{33}$ ergs per cubic centimetre; and that any streaming along magnetic lines of force is, in centimetres per second, one four-millionth of the number of c.G.s. units of intensity in the magnetic field.

\section{Part II. \\ Experimental Attempt to observe the Hypothetical Magnetic Ather Drift.}

16. During the years 1892-93, being urged thereto by certain mathematical theories, which are referred to and partly quoted in an Appendix in inverted order of date, I made a careful examination of the question of an ather flow along magnetic lines of force. This experiment has not been published, and in view of the above estimate its details ought to be recorded. As the result was negative I shall make the record as brief as possible. In July $1892 \mathrm{I}$ constructed four bobbins or frames of brass, each 44 centimetres long, with a core of elliptical section of great eccentricity, so that the major axis of its aperture was about ten centimetres and the minor axis about one centimetre. Each bobbin was provided with carefully glazed ends of optically worked plate-glass, that could be clamped on in a water-tight fashion, and with a means for supplying any desired liquid into the interior of the core. The surrounding brass-sheet walls of the core were double, so that between the core and the winding there was an annular space for water-jacketing, in which a current of cold water could be 
kept flowing, so as to screen the core from elevation of temperature due to the current in the exciting coil. The coil of wire wound on each bobbin had a resistance of $31 \mathrm{ohms}$ and consisted of seven thousand turns of silk-covered No. 18 wire ( .048 inch diameter), wound in 22 layers, with 7.2 turns to the centimetre in each layer ; thus giving a "gauss-gradient," or magnetic intensity, along the axis, of 200 times the amperes Howing in the wire.

With 110 volts applied, the current was $3 \frac{1}{3}$ amperes ; with 230 volts applied, which could be done by putting the town mains (at that time 110 volts) in series with a large storagebattery, the current was 7 amperes, measured by an amperemeter in both instances.

The corresponding intensities of field are

$\mathbf{H}=\left\{\begin{array}{r}670 \\ 1400\end{array}\right.$ c.G.s. lines to the square centimetre respectively.

17. To check this estimate, the four bobbins were filled with bisulpbide of carbon, placed all in a row, and supplied each with a current from the 110 -volt mains, the coils being electrically in parallel. Polarized sodium light was then passed through the set, and the plane of polarization was found to be rotated by the current $83^{\circ}$; of which $5^{\circ}$ was due to the glass, and $78^{\circ}$ to the bisulphide of carbon.

Hence the drop of magnetic potential between the ends of each coil comes out 29,000 gauss; according to Lord Rayleigh's estimate of the Verdet's constant for $\mathrm{CS}_{2}$ at $18^{\circ}$, viz., 042 minute of are for every G.G.S. unit of magnetic potential. The other estimate, from current and number of turns, under the same conditions, made the drop of magnetic potential $44 \times 670=29,500$ c.G.s.; which therefore agrees quite sufficiently well for the purpose.

18. All this is preliminary. In the actual experiment for trying the accelerating eff'ect of a magnetic field on light, the bobbins were not placed in a row, but were arranged round the sides of a square inscrihed at $45^{\circ}$ to the sides of another square or optical frame (about 1 metre in the side) of which the details are given in the Phil. Trans. 1893, pp. 757 and 761 , or more exactly in the plate 31 at the end of that volume (vol. 184) of the Phil. Trans.; since it was the same optical frame as was used for my rether convection experiments therein deseribed. (See also Preston's 'Light,' $\S 136$, and Proc. Roy. Inst. April 1, 1892.)

It is sufficient to say here that a parallel beam of light, bifurcated by semitransparent silvered glass at $45^{\circ}$, could be sent, by carefully adjusted mirrors, several times round the 
inscribed square, the two half beams going in opposite directions; they could then be re-united by the same semitransparent glass, and the interference bands observed in a telescope with a micrometer eyepiece.

During February 1893 a large number of experiments were made in this way. Sometimes the light was sent five or six times round, when only air was in the core, so that no coverglasses were required; sometimes water was inserted in the core, and then the light could be got three times round.

19. Sometimes, in the early trials, with a liquid in the cores, a darkening was seen when the current was put on, but never a shift of the bands; the darkening was capricious and could not always be got: it was suspected to be occasionally due to a slight shift of the bobbins through mutual magnetic attraction. Polarized light too was often employed; it emerged still fairly plane-polarized, if the plane of polarization was parallel or perpendicular to the plane of reflexion at the mirrors; otherwise of course it became elliptical. But still there was no shift of the bands.

20. In these early trials, however, I was not sure of seeing more than one-tenth of a band shift, and with bisulphide of earbon not more than one-fourth of a band.

The bisulphide was not often employed, since water was so mnch easier: water is not nearly so sensitive to changes of temperature. Moreover the strong refractive index of bisulphide of carbon made it difficult to keep the beans to their path, and prevent the bobbins from acting like prisms. But the chief difficulty lay in the extreme sensitiveness to changes of temperature, so that now the water-jacketing failed to protect the bisulphide of carbon from some incipient elevation of temperature, and the slightest change of that sort began to contort the bands and render them undecided; while, if the current was left on any considerable fraction of a minute, they became unrecognizable. The experiment with the bisulphide, therefore, could not be pushed to the same degree of completeness as in the case of air or water ; but while the observations lasted the effect was negative, and after each experiment several hours had to be allowed for the liquid to attain a uniform temperature again.

There was no special theoretical reason for supposing that the magnetic drift would be any stronger in bisulphide than in air, so this experiment was not persisted in.

If a transparent magnetic liquid could have been used, persistence would have been called for, but no such liquid is known; and though several attempts were made to use a solution of some salt of iron, the minute increase of Phil. Mag. S. 6. Vol. 13. No. 76. April 1907, $2 \mathrm{M}$ 
permeability, and therefore of etherial density, to be expected, was too insignificant to be of appreciable use. Besides, the oxidation of the sulphate, and the comparative opacity of the perchloride, made these liquids very difficult.

21. It is the experiments with air on which I place most reliance; and of these the best and final determinations were made six months later, immediately after receiving the following two letters from Dr. Larmor, which I am permitted to quote. The first letter seems to initiate the idea that I should try the effect of a longitudinal field on light; but I had already been trying it within the previous year-as just describedamong many other things in the course of my "aberration" work,

\section{Extracts from Letters from Dr. Larmor.}

“ 28 Oct., 1893.

"Your kindness in sending me a copy of your Aberration paper encourages me to ask you a question which now interests. me very keenly, namely, whether transmission along lines of magnetic force in air appreciably alters the velocity of light. I cannot discover that anyone has tried, but according to an ætherial theory which I have nearly finished writing out, and which covers, I fondly imagine, a very extensive ground, there ought to be such an effect. Perhaps you know, or could easily try with your machinery."

I suppose I replied that I had tried, and found nothing, but that I would repeat the trial more carefully if he would be satisfied with an experiment in air; for his next letter says :-

"1 Nov., 1893.

"I can correlate most things in one scheme if I am allowed that magnetic force is velocity of the æther. ... A magnetic field should carry the light along with it,--with what kind of velocity I have not yet formed an opinion; but it has nothing. to do with rotary effect, and should be of the same order in vacuum as in a mass."

22. In the experiment now to be described, as recorded in my laboratory note-book for Novernber 1893, the light was. sent four times round, in opposite directions, through the square of bobbins above described; the fact of four times being carefully tested, and sharp broad interference-bands being obtained. A fine micrometer cross-wire was set on the middle band, and watched, while 110 volts were applied to each coil, put on, off, reverse, on, off, \&c. No shift whatever was seen, either of the middle or any other band-no effect at all. Repeated carefully several times, and watched for so little as the $\frac{1}{10}$ th of a band shift, which previous experience 
had made me sanguine of seeing if it existed (see page 776, Phil. Trans. vol. 184). Could certainly have detected $\frac{1}{50}$ th of a band, probably $\frac{1}{100}$ th ; but nothing was seen.

23. The experiment was so good that it was decided to try a higher voltage; so, putting the town mains in series with a storage-battery, 230 volts were applied to each coil, giving a current of 28 amperes on the meter, or 7 amperes to each coil. The bands were again watched while the current was being put on and off and reversed, sometimes slowly and sometimes quickly, but I never saw the slightest motion of any kind. The current could not be left on very long, because of possible heat disturbance penetrating from the windings of wire: for the coils themselves naturally became hot, since 9 horsepower was being expended upon them, but the waterjacketing succeeded in keeping the cores quite cool.

24. Hence I conclude, with security, that if there is any æther-flow at all along magnetic lines of force, it bears to the velocity of light a ratio less than the ratio of $\frac{1}{50}$ th of a wavelength to 32 times the length of a bobbin. The number 32 being justified by the fact that there are four bobbins in optical series, and that the light is sent four times through each, in opposite directions simultaneously; so that the whole virtual length of path along which the slightest acceleration or retardation would have caused a shift of the bands, is $32 \times 44=1408$ centimetres. The ratio of a fiftieth of a wavelength to this distance is less than $10^{-9}$, so I conclude that any hypothetical æether drift, along a field of intensity 1400 c.G.S., is less than 30 centimetres, or one foot, per second; in other words, the ether flow is not as much as $\frac{1}{9}$ th of a millimetre per second, or say 16 inches an hour, for each c.G.s. unit of field intensity.

25. The total drop of magnetic potential applied was equivalent to $32 \times 60,000=$ nearly two million c.G.S., and this certainly did not hurry the light by so much as $\frac{1}{50}$ th of a wave-length. As a matter of fact $\frac{1}{100}$ th shift could certainly bave been seen, under the conditions; but I estimate $\frac{1}{50}$ th, to keep on the safe side.

So the deduction from this experiment was, either that the theory about longitudinal magnetic drift is erroneous, or else that the æether of space is at least eight times as dense as platinum.

26. That was the experimental conclusion thirteen years ago. I have now brought forward theoretical arguments which suggest that there is a sense in which what we call the 
density of æther may turn out to be fifty thousand million times the ordinary material density of platinum. In which case every cubic millimetre of space contains the equivalent of a thousand tons, and every part of it must be internally squirming with the velocity of light.

\section{APPENDIX A.}

QUOTATIONS FROM EARLIER WRITERS ON THE SUBJECT OF A HYPOTHETICAL MAGNETIC FLOW.

The experiments above described are referred to by Professor Larmor in his paper on "A Dynamical Theory of the Electric and Luminiferous Medium," in the Phil. Trans. for 1894, vol. 185, p. 778. A fairly high value of density for æther is there contemplated with equanimity; though it is pointed out that such density would require the æther to be absolutely stationary, except in a magnetic field, in order to avoid hydrodynamieal systems of force between moving bodies. But since everything tends to indicate that the ether is the most stationary substance in existence, at least as regards locomotion-probably the absolutely stationary thing, in so-far as the phrase means anything-this necessity involves no difficulty whatever.

27. To illustrate the theoretical bearing and expectation of a magnetic drift, it may be useful to make a few extracts:-

First I will quote from $\$ 73$ and 74 from the above 1894 paper of Prof. Larmor's (Phil. Trans. vol. 185, p. 773, \&e.).

"On the present theory, magnetic force or rather magnetic induction consists in a permeation or flow of the primordial medium through the vortex-aggregate which constitutes tho matter; apparently it has not been tried (see however \$ 81) whether light-waves are carried on by this motion of the medium and their effective velocity is thereby altered, as we would be led to expect. It has been shown, however, by Wilberforce that the velocity of light is not sensibly altered by motion along a field of electric displacement, so far negativing any theory that would connect electric displacement with considerable bodily velocity of the æther; and it has also been verified, by Lord Rayleigh, that the transfer of an electric current across an electrolyte does not affect the velocity of light in it. . . .

"As motion of the rether represents magnetic force, the fact that the magnetic permeability is almost the same in all sensibly nonmagnetic bodies as in a vacuum, must be taken to indicate that the ather flows with practically its full velocity in all such media, so that there is very little obstruction interposed by the matter; it follows that, in the motion of a body through the æther, the outside xther remains at rest instead of flowing round its sides. . . . .

" The notion of illustrating magnetic induction by the permeation of a fluid through a porous medium containing obstacles to its motion has been shown by Lord Kelvin to lead to a complete formal representation of the facts of diamagnetism." 
Also on pages 779 and 805 :-

"The residual forcive between bodies at rest in a field of ætherial motion, due to very slight defect of permeability, has already been shown, after Lord Kelvin's illustration, to simulate diamagnetism; and the fact that there exist no powerfully diamagnetic substances is so far a confirmation of the present hypothesis. 'The view that the magnetic field of a current involves only slight circulation of the fluid xther is also in keeping with the account which has been given $(\$ 46)$ of the genesis of such a field." ....

"The idea of representing magnetic force in the equations of electro-dynamies by the velocity of the electric medinm has been tried already, for example by Heaviside and by Sommerfeld, not to mention Euler. The objection however has been taken by Boltzmann and also by von Helmholtz, that it would be impossible on such a theory for a body to acquire a charge of electricity. A cardinal feature in the electrical development of the present theory is on the other hand the conception of intrinsic rotational strain constituting electric charge, which can be associated with an atom or with an electric conductor, and which cannot be discharged without rupture of the continuity of the medium."

28. G. F. FitzGerald also recognized the possibility or probability that the æther may have a high density; as is shown by the following quotation from his Helmholtz Memorial Lecture before the Chemical Society of London in 1896 (page 347 of his Collected Papers) while discussing the vortex theory of matter. After explaining that a liquid with inteuse internal movement, or kinetic structure, could propagate disturbances like light, provided there were no diffusion of the motion, he goes on to point out that "as vortices move of themselves freely and independently of the density of the liquid, there is no necessity for supposing that the density of the liquid is small. In fact, the simple theory would point to the conclusion that its density may be greater than that of platinum. This would require the energy per cubic centimetre of the turbulent motion to which the propagation of light is due to be very large indeed, approaching a million of millions of kilojoules, i.e. it would be equivalent to a million horse-power for 10 hours a day for nearly a month."

This guess at intrinsic ætherial energy makes it $10^{22}$ c.G.s. My paper has now estimated it as several thousand million times greater still.

29. At an earlier date, Mr. Heaviside's ideas on a magnetic analogue or model, based on fluid flow, may be typified by the following extract from a paper of his in the 'Electrician' for 1883. See 'Electrical Papers,' vol. i. pp. 270, 277.

"But by far the most interesting analogue is that presented by the motion of a perfect incompressible liquid. Let it fill all space (since the magnetic force of our rectilinear current extends without 
limit), and replace lines of force by lines of motion of the liquid, its velocity to everywhere correspond to the inagnetic force."

"The liquid within the cylindrical space corresponding to the wire is rotating, every particle of it, with the same angular velocity. The motion of the liquid outside the vortex, however, although the lines of motion are circles abont the vortex, is differentially irrotational. ....

"Current direction corresponds to the axis of a vortex, currentdensity to twice the angular velocity; vortex lines and tubes, analogous to current lines and tubes, are always re-entrant; the line-integral of magnetic torce about a current, measuring the amount of current, corresponds to the circulation of liquid about a vortex, being proportional to the angular velocity of the latter, etc.

"But, not to go into details, we will merely note, further, the analogue of the identity

$$
\Sigma \frac{1}{2} B_{1}^{2}=\Sigma_{2} A_{1} C_{1}
$$

[p. 244 ante]. Here, $B_{1}$ being the magnetic force of current $C_{1}$, whose potential is $A_{1}$, we have two expressions for the quantity that there is reason to believe is the kinetic energy of the system of magnetic force. Now, let $B_{1}$ be the relocity of a perfect liquid of unit density, then $\Sigma \frac{1}{2} B_{1}{ }^{2}$ is really the kinetic energy of the whole motion (sum of mass of each elementary portion $\times \frac{1}{2}$ square of its relocity). We have then the remarkable result that the kinetic energy of the moviug liquid can be expressed in terms of its angular velocity in those places where the motion is rotational, and of the corresponding vector-potential."

"Further, since if $C_{1}=0$ everywhere, making $\Sigma A_{1} C_{1}=0$, it follows that $\Sigma B_{1}{ }^{2}=0$, which (being the sum of squares) involves $B_{1}=0$ everywhere. That is to say, if there is no rotation there can be no motion of the liquid [except a uniform motion not ranishing at infinity]. Hence, if the liquid fills all space, its motion, whatever it may be, cannot be every where iruotational." ... .

" $\mathrm{V}^{2} \pi a^{3}$ is in fact the whole energy in the case of the moving liquid, including that of the sphere of same density."

30. But going still further back, to a time 35 years ago, Lord Kelvin's representation of a magnetic feld as a fuid-fiow may be illustrated by the foliowing quotations from a paper of his published in 1872; which is where we got the name 'permeability' from. See his 'Papers on Electrostatics and Magnetism,' pp. 581 and 568.

"It is convenient to attribute to the supposed liquid such a density that $4 \pi$ times the kinetic energy of liquid permeating a solid of unit permeability, reckoned per unit volume of the whole space occupied by porous solid and liquid, shall be equal to half the square of the 'flux'; the word flux being borrowed from Fourier's theory of the conduction of heat and adapted to the use we have to make of it."

"The whole strength of current in each circuit, reckoned in absolute electro-magnetic measure, is to be equal to the circulation 
of the fuid through that aperture divided by $4 \pi$. The resultant electro-magnetic force at any point will be numerically equal to the resultant fluid velocity at the corresponding point in the hydrokinetic system."

\section{Appendix B.}

\section{CONSIDERATIONS RESPECTING A ROTATIONATLY ELASTIC JETHER.}

On showing proof-sheets of the above to Prof. Tarmor, he favoured me, just before leaving for America, with a communication concerning the outstanding difficulties which had been felt by some mathematicians concerning the dynamical theory of the æther considered as a rotationally elastic medium; mentioning especially a critical remark of Lord Rayleigh, quoted by him in Phil. Trans. 1897 A. p. 230 , concerning what would happen if a set of electrons were to circulate continually round a ring fixed in space, so as always to affect and keep in rotation the same identical portion of æether, instead of constantly operating on fresh and fresh portions. I quote his letter:-

"If we imagine a permanent magnet fixed in aether (through which it is to be observed that the earth is moving at something like 20 miles per second), then the circulation of the æther round it which represents its magnetic field, though it is at each instant differentially irrotational, would ultimately bring each element of xther round again to its original position, yet with a finite resultant rotation acquired by it. This would mean that the circulation in the rther, considered as an elastic medium with simple rotational reaction stress, would in such a case ultimately choke jtself by becoming gradually held up against an accumulated rotational reaction. It would seem, therefore, that a magnet fixed in sether could not exist in absolute permanence. It is pointed out (loc. cit.) that this ultimate elastic choking can be delayed in time iudefinitely by taking the inertia of the æther very great, a hypothesis which there was no reason for excluding and which was accordingly then adopted.

"If the inertia of the ather were no greater than your own (itself immense) estimate, then the statement of the fourth section of your $\$ 14$ would seem at first sight to hasten up this time of a complete circulation of the æther, close around the moving electron there considered, and on the theory of a purely rotational æather the region very close around the electron would not be amenable to the ordinary circuital relations of electrodynamic theory, but would be governed by modified relations of form which might perhaps be worked out. 'There does not seem to be anything defnitely known that would absolutely exclude this way out of the difficulty; for, needless to say, the representation of an electron as a small conducting sphere surrounded by free rether and with a charge on it, deformable or not, is merely an illustration; and the interesting calculations that can be made about it must not obscure the fundamental fact that it implies a reversal of the order of ideas, 
which was to explain electricity by electrons and not to explain electrons by electricity. The only thing experimentally known in this domain seems to be Kauffmann's result, which requires that an electron is some structure expressible analytically as formally equivalent to a spherical aggregation of 'electric charge' with invariable volume relations, but with 'density of charge' that may vary, according to any law whatever, in terms of the distance from the centre. For other electrodynamic purposes this structure can be treated simply as if it were an aggregate charge concentrated in a point.

"The case has here been put at its worst, and this aspect of it, along with unusual features in the phenomena of mechanical reaction, has in fact led M. Poincaré * and others following to ascribe insuperable difficulties to a rotationally elastic representation of the ather, notwithstanding the direct and natural representation which it gives of the field of an electron, as a self-locked stress-system keying on to it.

"But as I have been in the habit of explaining in academic lectures for some years, though I have not found time to explain the point in print, the case is far from being so hopeless. It may or may not be true that a permanent magnet (so-called), if absolutely at rest in æother, would through lapse of time have its magnetic field gradually choked: there is no direct means of knowing, so long as we must rush through space along with the earth at 20 miles per second. Take, however, your own supposition of an electron rushing through space and having a circulating movement of wther relative to itself which is of the same order of velocity close up to the electron as its velocity of translation. If this were steady actual circulation of the xther, instead of merely apparent circulation relative to the moving electron, such an idea of an electron would be blankly impossible on a rotationally elastic theory. But on consideration it will appear there is in fact no cumulative circulation at all. The electron is constantly shedding: off and leaving behind it, as a sort of wake in the stationary æther, these beginnings of circulatory motion which constitute its magnetic field, and thus the choking process never gets a start: in fact in the nature of the case it can never get a start (if the inertia of wther is sufficiently great), for the very essence of a magnetic field is that it is a disturbance which arises from the electrons moving on, and thus of necessity leaving it to collapse behind them.

"Problems in hydrodynamics of the general character suggested by these remarks have been dealt with long ago by Clerk Maxwell ("On the Displacement in a Case of Fluid Motion," Proc. London Math. Soc. iii., or Scientific Papers, vol. ii. pp. 208-214). He there considers the special case of a circular cylinder progressing with uniform velocity through quiescent frictionless fluid: the

* See the discussion at the end of the second edition of his Electricité et Optique, where the theory was by misunderstanding, doubtless due to its mode of presentation, contrasted with that of Lorentz, the two being in fact formally equivalent, as is now recognized. 
stream lines relative to the cylinder are, as is well known, steady and beautifully simple; but the actual path of the particles of this fluid as they are put into motion by the neighbourhood of the cylinder and finally left at rest in a displaced position after the cylinder has passed on, is a very different affair, as may be seen from the traces given in Maxwell's paper.

"As your proof-sheets and queries have forced me back on this subject, I hope to arrange for obtaining some early investigation of the corresponding magnetic problem, viz. the displacement and strain in a rotational ather due to an electron rushing through it, on the usual kind of working hypothesis that the electron is representable as if it were a small charged body."

P.S. "On second thoughts the total displacement of position of an element of æether at $\mathbf{P}$ due to an electron $e$ passing near it at distance $h$ with uniform velocity $v$, is readily worked out. For it

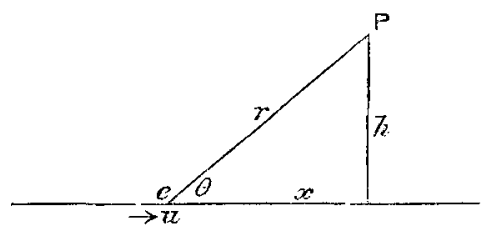

takes place around the axis of motion of the electron; and, if velocity of æther $=k \times$ magnetic force, we have

$$
\begin{aligned}
\text { arc of displacement } & =z \int \frac{t u \sin \theta}{r^{2}} d t, \text { where } u d t=d x \\
& =k \int_{\int^{2}}^{e} \frac{e}{h} \sin \theta \cdot d \theta \\
& =\frac{2 k e}{h}, \quad \text { independent of } u ;
\end{aligned}
$$

and the angle turned through in traversing this arc $=\frac{2 k e}{h^{2}}$.

"This rotational total displacemement of the element of æther, arising from a motion differentially irrotational, is greatest when $h$ is smallest. When $h$ is but little greater than the effectivo radius $a$ of the electron, the analysis is not adequate, for the electron cannot then be treated as a point.

"It will, however, still give the order of magnitude of the rotation: so putting $e=10^{-21}, h=a=10^{-14} \mathrm{~cm}$. it gives $2.10^{7} k$ radians *. If this is to be small, $k$ must be at least of the order $10^{-9}$; so that unit c.G.s. magnetic field would be represented by an rether-velocity of not more than $10^{-9} \mathrm{~cm}$. per sec. This would require the density of æther to exceed say $10^{16}$. But observe that the total arcual displacement, being proportional to $h^{-1}$, can mend itself (as is of course

* The numerical index of each of these estimates would by me be reduced by unity; but it does not matter for the main lines of the argument.-O.L. 
known) by an irrotational motion of the other having velocitypotential of form $\mathrm{A} \log h$; thus in its aggregate amount it involves no elastic reaction, and disappears spontaneously by irrotational flow when the electron has passed on. Thus, even with much lower effective inertia of the xther, the passage of the electron would not involve any permanent elastic dislocation in its surroundings, though very near it there might be increased elastic reaction equivalent to increase of its effective size: it being understood that so long as we do not know how the core of the electron is keyed together, the nature of its own penetration through æther-whether it be analogous to the motion of a vortex ring or otherwise-is beyond our cognisance, and must remain a matter of immediate hypothesis."

From this note a deduction at once follows, thus :-

'The angular displacement at any distance $h$ from path of electron, after it has passed, is $\phi=2 k e / h^{2}$; so the linear velocity of rether circulation at that place, while the electron is passing, is

$$
w=k \mathrm{H}=\frac{k e u}{h^{2}}=\frac{1}{2} \phi u .
$$

Everywhere, except close to an electron, $\phi$ is extremely small; but I see no reason for attributing to $\phi$ any but a finite value $(i, e$. something comparable to a radian) quite close to an electron. If that is permissible, the above little calculation is confirmatory of the hypothesis that $w_{0}$, the rotation speed round the equator, is of the same order as $\imath$, the translation speed of an electron. But Larmor supposes that the angular shift must be small everywhere. In that case $w_{0}$ would be less than $u$, and the estimate of ætherial density, together with the values of the other extraordinarily large quantities in this paper, would have to be correspondingly increased.

\section{APPENDix C.}

The following brief note from Professor J. J. Thomson is of special interest:- -

"Cavendish Laboratory, $13 \mathrm{March}, 1907$.

"Many thanks for letting me see the proof of your paper. I have beld for some time the view that the mass of bodies is the mass of wther they carry along with them, and that this mass can be carried along by the lines of electric force as well as the bodies these lines connect. The amount carried by the small negative ond is small compared with that carried by the lines of electric force, while the amount of the other carried by the large positive end exceeds that carried by the lines; hence the mass of the corpuscle seems wholly electrical, while that of the atom does not. I think, however, both masses are identical in origin, being masses of portions of æther. I went into this view at some length in my 'Electricity and Matter,' and have been confirmed in it by later consideration. of course it requires a very dense æther."

It is satisfactory thus to find a fair consensus of leading opinion in favour of some such proposition as $I$ have endeavoured to argue out, and make more precise, in the above paper. 